\title{
Development of the NIH Patient-Reported Outcomes Measurement Information System (PROMIS) Medication Adherence Scale (PMAS)
}

This article was published in the following Dove Press journal: Patient Preference and Adherence

\author{
John Devin Peipert $\mathbb{D}^{1, *}$ \\ Sherif M Badawy,* \\ Sharon H Baik (ID) \\ Laura B Oswald' \\ Fabio Efficace ${ }^{3}$ \\ Sofia F Garcia (D) ${ }^{\prime}$ \\ Daniel K Mroczek (D) \\ Michael Wolf ${ }^{4}$ \\ Karen Kaiser ${ }^{1}$ \\ Betina Yanez' \\ David Cella $\mathbb{D}^{\prime}$
}

'Department of Medical Social Sciences, Northwestern University Feinberg School of Medicine, Chicago, IL, USA;

${ }^{2}$ Division of Hematology, Oncology and Stem Cell Transplant, Ann \& Robert H. Lurie Children's Hospital of Chicago, Chicago, IL, USA; ${ }^{3}$ Italian Group for Adult Hematologic Disease (GIMEMA), Health Outcomes Research Unit, Rome, Italy; ${ }^{4}$ Department of Medicine, Division of General Internal Medicine,

Northwestern University Feinberg

School of Medicine, Chicago, IL, USA

*These authors contributed equally to this work

Correspondence: John Devin Peipert Email john.peipert@northwestern.edu

\begin{abstract}
Poor medication adherence is associated with reduced drug effectiveness, poor health-related quality of life, increased morbidity and mortality, and increased healthcare utilization and cost. Including the patient's voice is essential in understanding barriers to adherence. Useful patient-reported adherence measures are brief, inexpensive, noninvasive; can indicate barriers to adherence; and can be incorporated in electronic health records. The NIH Patient-Reported Outcomes Measurement Information System $\left(\right.$ PROMIS $^{\circledR}$ ) includes high-quality, freely available patient-reported measures covering many important constructs in patient-centered research but does not include a medication adherence measure. To fill this gap, we developed the PROMIS Medication Adherence Scale (PMAS) using the rigorous PROMIS instrument development guidelines. To develop the PMAS, we first conducted a review of the reviews, which enabled us to identify content areas relevant to medication adherence behavior. Then, we conducted qualitative research to elicit patients' views of and experiences with medication adherence. This process identified the following important content areas to guide item writing: extent medication is taken, knowledge of medication regimen, beliefs about medication, remembering to take medication, skipping due to side effects, skipping due to feeling better, and cost of medications. Based on the results of these activities, we wrote items and aimed to retain 1-2 items per content area. The final item set included 9 total adherence items, which were then refined through intensive comprehension and translatability review, as well as cognitive interviews. Future steps include testing the PMAS's validity.
\end{abstract}

Keywords: medication adherence, patient-reported outcome, PROMIS

\section{Plain Language Summary}

This paper details the development of a new patient-reported measure of medication adherence, the Patient-Reported Outcomes Measurement Information System (PROMIS ${ }^{\circledR}$ ) Medication Adherence Scale (PMAS). The PMAS was developed using a rigorous approach involving extensive patient input. These methods included a systematic review of reviews that summarized and rated patient-reported medication adherence measures, identifying the most important content featured in existing medication measures, and interviews with patients to determine their views of important medication adherence barriers and concerns. Based on the information collected, we wrote new items to be included in the PMAS, which we then piloted tested with patients to come to a final item set. With 9 medication adherence items, the PMAS is brief and covers multiple potential reasons patients with various chronic health conditions may not adhere to their medications. The PMAS will be freely available 
and is expected to be useful in clinical assessment and research. Next steps include validation of the PMAS with multiple clinical patient populations.

\section{Introduction}

Medication adherence is a key component of effectively managing illnesses and achieving target outcomes in acute and chronic health conditions. ${ }^{1-9}$ Poor medication adherence is a common, pervasive, and long-standing healthcare problem. It is considered by the WHO as a "worldwide problem of striking magnitude." ${ }^{, 7}$ Patients miss medication doses frequently, particularly patients with chronic health conditions. ${ }^{7}$ In addition, patients often err in taking their medications as prescribed, and dosing errors are very common among older adults especially. ${ }^{10,11}$ Moreover, $20-25 \%$ of patients never fill their prescriptions, and about $20 \%$ take "drug holidays" (ie, consciously not taking their prescribed medication for a period of time) after filling the prescriptions. ${ }^{6,7,12-15}$ While there may be multiple causes of poor medication adherence, reasons for not being fully adherent to a given regimen can be broadly grouped into: 1) intentional (ie, a conscious decision to not take their prescribed medication; eg, negative perceptions of medication or its side effects); or 2) non-intentional (eg, dosing errors, forgetfulness, carelessness). ${ }^{6,7,9,16,17}$

Regardless of the cause of poor medication adherence, it has been associated with worse health outcomes, impaired health-related quality of life (HRQOL), higher symptom burden, higher mortality rates, and increased healthcare utilization with unnecessary cost $(\$ 100$ billion in the United States [US] and $€ 25$ billion in the European Union), as well as lower personal and work productivity and frustration among patients and clinicians. . $^{67,14,15,18-24}$ Given the documented negative consequences of poor medication adherence, routine assessment of patients' adherence levels and related challenges is key to identifying actionable barriers and optimizing treatment outcomes. Thus, there is an essential need for a standardized, brief, and validated patient-reported medication adherence measure with appropriate psychometric properties for use with various pediatric and adult patient populations. Such a measure would help to identify patients at high risk for poor medication adherence and possible barriers to adherence behaviors, as well as inform the development of individually tailored interventions that promote medication adherence and optimize health outcomes in different patient populations. $^{25,26}$
Patient self-report using standardized survey measures is among the most commonly used tools for measuring medication adherence and potentially offers the most efficient, lowest-cost and easiest-to-implement approach to screen for adherence behavior in research and real-world settings. ${ }^{27-29}$ Patient-reported medication adherence measures assess patients' medication-taking behaviors (eg, taking medication as prescribed, refilling prescriptions on schedule), barriers to medication adherence (eg, adverse side effects), and beliefs associated with medication adherence (eg, necessity of medication). Previous reviews of patient-reported medication adherence measures show that they are often reliable and valid, with some variation, ${ }^{30,31}$ and they are feasible to administer as part of clinical patients interviews. A notable advantage of patientreported medication adherence measures is their ability to identify reasons for medication non-adherence. Many of the patient-reported medication adherence measures focus on barriers to adherence such as forgetting, lack of knowledge or confusion about the regimen, intentionally not taking a medication, and other issues that can help identify the cause for poor adherence among individual patients. ${ }^{32,33}$ This actionable information can then be used to tailor approaches to address poor medication adherence among individuals. ${ }^{27}$ The ability to identify reasons for non-adherence make self-report measures useful in both clinical practice, as well as research.

The Patient-Reported Outcomes Measurement Information System (PROMIS ${ }^{\circledR}$ ) Medication Adherence Scale (PMAS) was developed to improve self-reported assessment of medication adherence. The PROMIS is a National Institutes of Health (NIH) roadmap initiative to improve the measurement of various domains of HRQOL and symptoms and develop a system of psychometrically sound, flexible, and universal measures of patient-reported outcomes (PROs) relevant for a wide range of diseases. Using rigorous methods, over 100 PROMIS measures have been developed and validated, spanning various HRQOL domains (www.healthmea sures.net), and all PROMIS measures are widely available and freely accessible to researchers and clinicians. The objective of this paper is to report the process of developing the PMAS. ${ }^{34-40}$ Consistent with prior PROMIS measure methods, the PMAS was developed with both patient and clinician input and involved an iterative process consisting of a review of the literature, content area analysis, concept elicitation, new item writing, and item review and revision (Figure 1). 


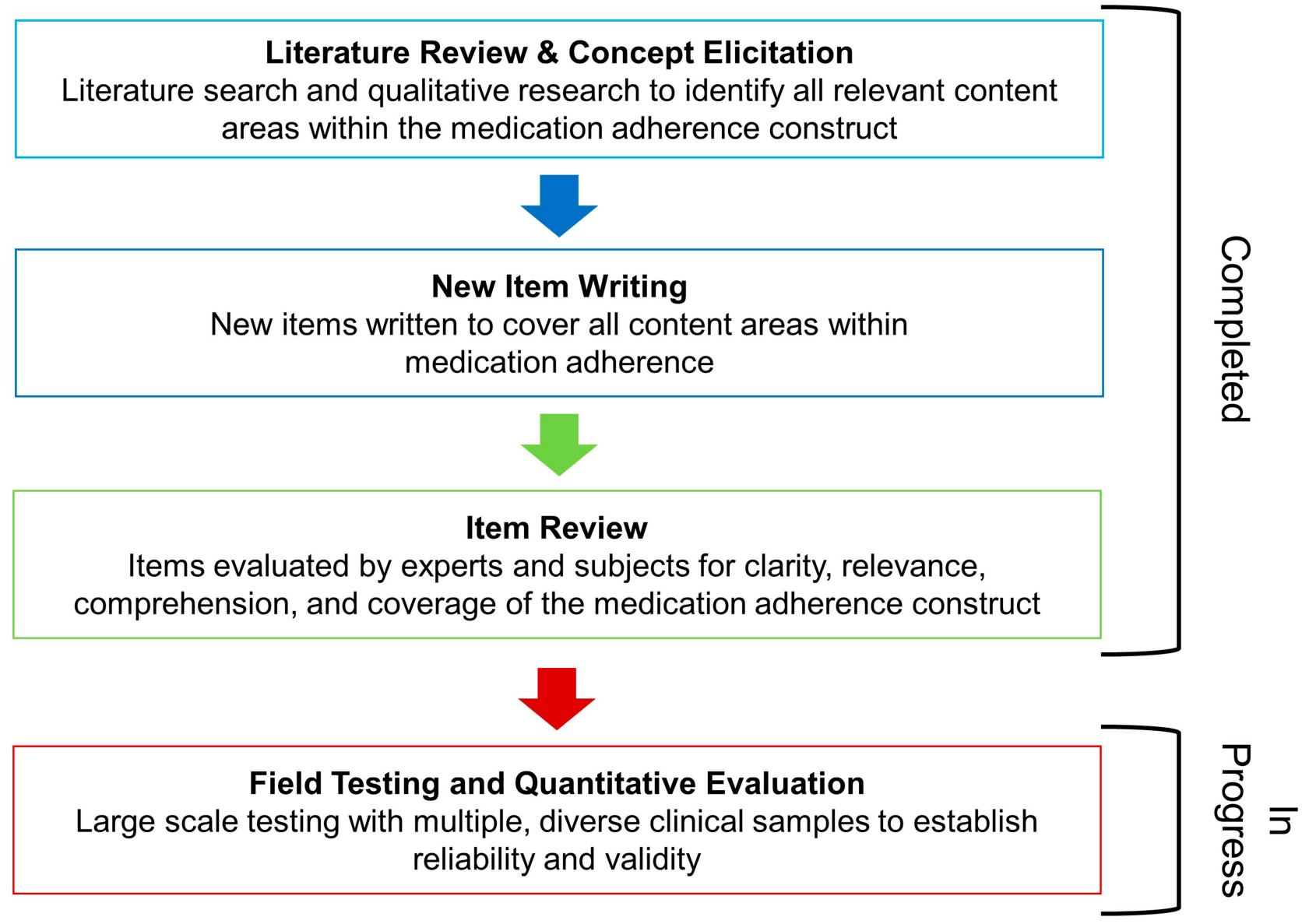

Figure I PMAM development process.

\section{Methods}

\section{Review of Literature}

Since there are already several recent systematic reviews of medication adherence measurement, we conducted a "review of the reviews." Structured searches of the literature for reviews in Pubmed were conducted using keywords "(medication adherence) AND (conceptual model)" and "(medication adherence) AND (conceptual framework)" in July 2018. The results and conclusions of these reviews were then summarized and synthesized to identify content areas and methodological concerns to address in the creation of the PMAS.

\section{Content Area Analysis of Legacy Measures}

Based on the results of our literature search, legacy measures were identified for inclusion in a conceptual analysis to further narrow PMAS content areas. The objective of this analysis was to identify commonly-used, patient-reported medication adherence measures and determine the most common constructs covered in these measures. This analysis was performed by five team members (measurement scientists) who separately coded the individual items of each legacy measure to categorize each item into separate content areas.

The analysis was performed according to the following steps. Step 1: First, legacy measures were identified in the review of reviews and selected for consideration by a team of 5 measurement scientists using multiple criteria, including focus on oral medications, psychometric properties (emphasizing predictive validity, criterion validity, sensitivity, and specificity), number of citations in the scientific literature, and whether it was generic vs health conditionor treatment-targeted. The group considered and discussed these criteria, then selected a set of measures that had evidenced good measurement properties and that were commonly-used, and therefore would likely represent constructs sought after by researchers and clinicians. In addition, a diversity in the measures' focus and types of items featured was sought after. 
Step 2: Next, after selecting items, we compiled and numbered a list of all the items across the selected legacy measures, determining if any were not unique. Step 3: Then, a context coding process was undertaken using a hybrid inductive-deductive strategy. An initial set of codes was created by two team members after drawing themes from the first three measures reviewed. Each code represented a unique content area. The content areas identified through the review of reviews were also crosschecked for consistency with themes emerging from the initial analysis of legacy measures. Next, the remaining legacy measures were analyzed using the initial set of codes and codes were added, as needed. Coding discrepancies were brought to a monthly team meeting to be discussed and resolved by consensus. After coding each item, we recorded the frequency of each code. Content area codes occurring five times or more were selected for inclusion in the PMAS.

\section{Concept Elicitation Study}

Additional content areas relevant to medication adherence were identified through qualitative interviews with patients. Specifically, we conducted a study to elicit facilitators and barriers to medication adherence among Latina breast cancer survivors in English and Spanish. Inclusion criteria were: diagnosed with early-stage breast cancer but without prior history of breast cancer, initiated endocrine therapy, completed between 6 months and 5 years of breast cancer treatment, self-identified as Hispanic/Latina, and English- or Spanishspeaking. Participants were classified as non-adherent to endocrine therapy if they endorsed non-adherence on the Revised Medication Adherence Questionnaire. Breast cancer patients on endocrine therapy are an appropriate clinical population for medication adherence measure development because nonadherence in clinical settings among this population is common and is often driven by multiple causes (eg, beliefs, cost). ${ }^{41}$

Individual interviews were audio-recorded, transcribed, and de-identified prior to analysis. Transcripts in Spanish were translated into English by at least two bilingual members of the research team and checked for accuracy by a third bilingual member of the research team. Qualitative data was analyzed for thematic content by two coders using NVivo software. First, in an initial coding meeting, the research team and a community consultant identified and listed potential themes of interest. After coding rules were established and coding definitions were created, a code book was developed. Each transcript was independently coded by two coders. The objective of these analyses was to reach conceptual saturation, at which point no new themes were identified.

\section{New Item Creation}

To create a new pool of items to draw from for the PMAS, we wrote new items de novo; no items were drawn from the legacy measures. Multiple candidate items were written for each content area identified in the review of the reviews, analysis of legacy measures, and concept elicitation study. The initial set of items was refined through preliminary review by the measurement development team and by a professional patient-reported measure translator (PROMIS linguist) who assessed translatability of the items. The initial pool of items was reduced and refined by eliminating items that were deemed unclear, were double-barreled, used vague terms or slang/jargon, and captured the content area of interest in a less concise or simple way compared to the other candidate items.

\section{Cognitive Interviews}

After the initial set of items was created, we conducted cognitive interviews to determine whether the items were understandable and whether they captured their intended meaning. We recruited patients with a variety of chronic illnesses to participate in the cognitive interviews from 3 ongoing studies at Northwestern University, one with breast cancer survivors (PI: Yanez), one with diabetes patients (PI: Wolf), and one with kidney transplant recipients (PI: Wolf). Participants from each of these studies were called and asked to them to participate in the ancillary cognitive interview study. Once five participants for the cognitive interviews had been found, recruitment to the cognitive interview study was ceased.

A semi-structured interview guide was used. First, we collected data about patients' health history and medications used to treat their primary chronic illness. Then, we administered the draft PMAS. After completing the PMAS, patients were asked a series of questions about each item (eg, "How would you say this question in your own words?"; "Is this question relevant to your experience or your medicine?", "What did you think about when you answered the question?"). Notably, we asked whether the PMAS items covered all relevant aspects of patients' experiences with their medications and if there were important questions missing from the PMAS. In addition, key phrases were probed on to ensure their meaning was clear. The cognitive interview results were aggregated and analyzed to generate recommendations for changes in item wording. 


\section{Results}

\section{Review of Reviews}

Seventy-seven unique papers were identified. Of these, eight papers were relevant to the topic. ${ }^{27,30,42-47}$ All but one was general in its scope in terms of the patient populations addressed. Specifically, the review by Murray et $\mathrm{al}^{47}$ addressed issues of adherence in older adults but still covered multiple medical conditions. In general, papers were considered irrelevant because they were not review articles, they were narrowly focused on a single clinical population, or they were viewpoints or strictly theoretical. In addition, a relevant review paper by Voils et al missed in our systematic review was found afterward and included. $^{48}$

The reviews pointed to several important content areas to consider for the PMAS, including the extent of medication adherence, multiple barriers to adherence, beliefs about adherence, and knowledge of the regimen. For example, Stirratt et al's review ${ }^{46}$ identified "extent of adherence" as one of several foundational content areas relevant to the behavior of medication adherence, and is most closely linked to the actual definition of medication adherence. This content area is captured with items asking about the number of pills or doses taken or missed, recalling over a pre-specified time (eg, past 7 days). In addition, this content area is assessed in $70 \%$ of patient-reported medication adherence measures. ${ }^{30}$ In Nguyen et al's review, $70 \%$ of measures $(n=30)$ asked about barriers or determinants of medication adherence, and $16 \%$ asked about medication beliefs.

Critically, Voils et al pointed to the importance of distinguishing between patient-reported adherence items that are causal indicators and effect indicators. ${ }^{48}$ In this context, items that represent the extent of adherence are effect indicators, since they reflect the results of medication adherence. On the other hand, items representing reasons for non-adherence are causal indicators, since they represent causes of medication adherence. In their review, Voils and colleagues point to the potential to improve patient-reported adherence measurement and better represent the construct by separating extent of medication adherence and reasons for medication adherence into distinct scales, each supported by different methods.

Culig et al's review commented on the importance of both intentional (eg, refusal to take) and unintentional (eg, forgetting) barriers or reasons for non-adherence. The relevance of both types of barriers for older adults was also highlighted by Murray et al. ${ }^{47}$ These authors called attention to issues related to unintentional non-adherence like forgetting medications or confusion about the regimen, which is sometimes linked to cognitive issues, and general inability to manage and self-administer medications. Likewise, there may be intentional resistance to adherence. This resistance may be tied to confusion around the role and purpose of medications, especially when there is polypharmacy.

In addition to useful information about priority content areas, the reviews also commented on the most appropriate response options for items in patient-reported adherence measures. In Nguyen et al's review, of those asking about extent of medication taking, $67 \%$ specified a recall period (eg, "In the past 7 days ..."). Recall period time frames ranged from 1 day to 12 months across legacy measures. The reviews found little comparative research of differing response option types. ${ }^{27,42}$ However, it is noted that the use of dichotomous response options in order to easily calculate a "percent adherent" score may be oversimplified and result in a less-useful contribution of patient-reported medication adherence measures, at least in the context of routine clinical monitoring. Nonetheless, the review by Lavsa et al emphasized the importance of easy scoring during clinical encounters. ${ }^{45}$ They found that only a few of the available measures facilitated handscoring that could easily be implemented in clinic.

\section{Content Area Analysis of Legacy Measures}

From our review of reviews and through group consensus, we identified and prioritized ten commonly-used, legacy patientreported measures of medication adherence. (Table 1) In general, these measures balance a diverse selection of measures with strong measurement prosperities, very frequent use in research, and generic vs disease- or treatment targeted orientation. These measures included the Adherence Starts with Knowledge-12-Item (ASK-12), ${ }^{49}$ the Medication Adherence Scale (MAS), ${ }^{50}$ the Adherence Self-Report Questionnaire (ASRQ), ${ }^{51}$ Adherence Evaluation of Osteoporosis Treatment-12-Item (ADEOS-12), ${ }^{52}$ an HIV-targeted singleitem measure, ${ }^{53}$ the Simplified Medication Adherence Questionnaire (SMAQ) ${ }^{54}$ the Immunosuppressant Therapy Adherence Instrument (ITAS) ${ }^{55}$ the Adherence to Refills and Medication Scale (ARMS), ${ }^{56}$ the 4-item Morisky Medication Adherence Scale (MMAS-4), ${ }^{32}$ and the 8-item MMAS (MMAS-8).$^{33}$ Inter-rater agreement was reached for 
Table I Legacy Adherence Measures Reviewed for Content

\begin{tabular}{|c|c|c|c|}
\hline Measure & $\begin{array}{l}\text { Number of } \\
\text { Items }\end{array}$ & Population & Citation \\
\hline Adherence Starts with Knowledge-12-Item (ASK-I2) & 12 & Generic & Matza et al $2009^{50}$ \\
\hline Medication Adherence Scale (MAS) & 18 & Generic & Wu et al $2008^{51}$ \\
\hline Adherence Self-Report Questionnaire (ASRQ) & 6 & Persons with hypertension & Zeller et al $2008^{52}$ \\
\hline Adherence Evaluation of Osteoporosis Treatment-12-Item & 12 & Persons with osteoporosis & Breuil et al $2012^{53}$ \\
\hline HIV-targeted single item measure & 8 & Persons living with HIV & Feldman et al $2013^{54}$ \\
\hline Simplified Medication Adherence Questionnaire (SMAQ) & 6 & Persons living with HIV & Knobel et al $2002^{55}$ \\
\hline Immunosuppressant Therapy Adherence Instrument (ITAS) & 4 & $\begin{array}{l}\text { Organ transplant recipients on } \\
\text { immunosuppressants }\end{array}$ & Chisholm et al $2005^{56}$ \\
\hline Adherence to Refills and Medication Scale (ARMS) & 12 & Generic & Kripalani et al $2009^{57}$ \\
\hline Morisky Medication Adherence Scale-4 Item (MMAS-4) & 4 & Generic & Morisky et al $1986^{33}$ \\
\hline Morisky Medication Adherence Scale-8 Item (MMAS-8) & 8 & Generic & Morisky et al $2008^{34}$ \\
\hline Voils Extent and Reasons for Medication Adherence & 28 & Generic & Voils et al $2012^{58}$ \\
\hline
\end{tabular}

the coding of the vast majority of items ( $\mathrm{n}=83,92.2 \%)$. For cases in which consensus was not reached $(\mathrm{n}=7,7.8 \%)$, coding was rectified via group review and discussion. After the completion of the review of these 10 legacy measures, an additional legacy measure was identified through further review and added to the content analysis of items. This was the measure created by Voils et al focusing on extent and reasons for medication non-adherence. ${ }^{57}$ Across these 11 measures, there were a total of 118 unique items assessing medication adherence. Table 2 displays the unique content areas evaluated across the 11 legacy measures.

These legacy measures varied in the content areas they covered, length, and target clinical populations used. For example, the $\mathrm{ARMS}^{56}$ and ASK- $12^{49}$ are generically targeted for patients with chronic diseases. The ARMS and ASK-12 are multidimensional and consist of 12 items with responses rating the level of agreement and frequency of behaviors using a 4- or 5-point Likert type scales. ${ }^{49,56}$ Specifically, the ARMS assesses adherence to taking medications and refilling prescriptions, ${ }^{56}$ and the ASK-12 has three subscales for medication-taking behaviors, inconvenience/forgetfulness, and health beliefs. ${ }^{56}$ Notably, the Voils measure separates extent of medication adherence and reasons for medication adherence as two separate constructs, represented by two separate scales. ${ }^{57}$ This distinction is based on this group's insight that reasons for medication adherence items are causal indicators and extent of medication adherence items are effect indicators; they have argued that scales constructed based on these two types of items require different methodological approaches. Other legacy measures were targeted to specific disease groups. For example, the ADEOS- $12^{49}$ specifically frames each question for the respondent's "osteoporosis medication," and seeks to capture information on treatment and patient attributes relevant to this disease. Similarly, the 6-item ASRQ ${ }^{51}$ and 4-item ITAS ${ }^{49}$ assess overall adherence to blood pressure or immunosuppression medications, respectively. For the PMAS, content

Table 2 Frequency of Items Assessing Content Areas Associated with Reasons for Medication Adherence and Non-Adherence Across Legacy Measures

\begin{tabular}{|l|l|}
\hline Content Area & Item Frequency (\%) \\
\hline Extent of missing doses & $15(12.7)$ \\
Planning to take medication & $15(12.7)$ \\
Extent of adhering to medication & $11(9.3)$ \\
Forgetting to take medication & $11(9.3)$ \\
Experiencing side effects of medication & $10(8.5)$ \\
Beliefs about medication & $9(7.6)$ \\
Feeling better & $7(5.9)$ \\
Knowledge about medication/regimen & $5(5.6)$ \\
Complexity of regimen & $4(3.3)$ \\
Unintentional non-adherence & $4(3.3)$ \\
Inconvenience & $4(3.3)$ \\
Cost of medication & $4(3.3)$ \\
Worry & $4(3.3)$ \\
Instruction Issues & $3(2.5)$ \\
Intentional non-adherence & $2(2.2)$ \\
Motivation to take medication & $2(2.2)$ \\
Self-efficacy to adhere & $2(2.2)$ \\
Social support (or lack of) & $2(2.2)$ \\
Shared decision-making & $1(1.1)$ \\
Short supply & $1(I .1)$ \\
Habit formation & $1(1.1)$ \\
Uncoded & $1(1.1)$ \\
\hline Note: There were II unique &
\end{tabular}

Note: There were 118 unique items across the II legacy measures. 
areas represented by five or more unique items across the legacy measures were retained for item development. The retained content areas were: extent of missing doses, forgetting to take medication, beliefs about medication, extent of adhering to medication, planning to take medication, experiencing side effects of medication, feeling better, and knowledge about medication/regimen. Content areas that occurred less than five times were complexity of regimen, overall unintentional non-adherence, inconvenience, cost of medication, overall intentional nonadherence, motivation to take medication, self-efficacy to adhere to medications, social support (or lack thereof), shared decision-making, short supply, and habit formation.

\section{Concept Elicitation Results}

The sample for individual interviews included 31 Latina breast cancer survivors who had initiated endocrine therapy (a medication that is taken daily for 5-10 years). Participants were an average of 55 years old, and $61 \%$ of participants had a high school degree or less. Less than half of participants (48\%) had a diagnosis of stage I or II breast cancer, with $84 \%$ having received radiation therapy and $58 \%$ having received chemotherapy. The rate of nonadherence to endocrine therapy was $39 \%$.

Regarding the thematic analysis, there was coder agreement of $\geq 70 \%$ for all codes. Common themes among non-adherent participants were of particular interest, since these were likely to indicate barriers to medication adherence. The most common themes among this group were the cost of endocrine therapy, low selfefficacy, managing side effects, being unsure of how to take endocrine therapy, and being unsure of the purpose of endocrine therapy (Figure 2). Among patients classified as adherent, the cost of endocrine therapy was also a common theme along with the ability to manage side effects.

\section{Item Pool for the PMAS}

Using the results of the review of reviews, legacy measure analysis, and concept elicitation study, we identified multiple content areas to guide the writing of items for the PMAS. After selecting the content areas that occurred five times or more in the legacy measure analysis, content areas that had significant overlap were collapsed together (eg, extent medication taken and extent missed doses). We then compared this set of content areas to those identified in the concept elicitation study. These were mostly wellaligned, with a few exceptions. Specifically, cost of medications and self-efficacy to adhere to medications were both given high priority in the concept elicitation study. However, only cost of medications was included in the PMAS, as there is already a PROMIS measure for assessing self-efficacy (PROMIS Self-Efficacy for Managing Chronic Conditions - Medications and Treatments). ${ }^{58}$

The key content areas of medication adherence used to guide the development of the PMAS item pool were extent of missing doses, planning to take medication, extent of adhering to medication, forgetting to take medication, experiencing side effects of medication, beliefs about medication, feeling better, knowledge about medication/regimen. After selecting those key content areas, up to five candidate items were written to represent each content area. Then, item sets were reviewed by the measure development team and iteratively refined. The pool of items was reduced by narrowing down the items that: 1) best represented the content area of interest; 2) were most concise and simple in structure; and 3) were most understandable. Our aim was to retain one to two items per content area. Where possible, each item was written with a positive orientation to capture pro-adherence behaviors rather than non-adherence. For example, instead of asking about forgetting to take medications, we asked about remembering to take medications.

After selecting the best candidate items, the resulting set was comprised of nine total medication adherence items. This item set included one item to represent the "extent medication taken" content area, two items representing "knowledge of the medication regimen," two items representing the "beliefs about medication," one item representing "remembering to take medication," one item representing "skipping due to side effects," one item representing "skipping due to feeling better," and one item representing "cost of medications." All of the items were designed to be rated using a 5-point ordered categorical response scale, which is typical for PROMIS measures. The four items reflecting knowledge of regimen and beliefs about medication were given agreement-based response options ranging from (1) "Strongly Disagree" to (5) "Strongly Agree." These items were not given a context or recall period. The remaining items all assumed frequency-based response options ranging from (1) "Never" to (5) "Always." These items use a 7-day recall period (ie, "In the past 7 days ..."), which is standard for PROMIS measures. After the items were selected and the response options and context or recall periods were specified, a formal 


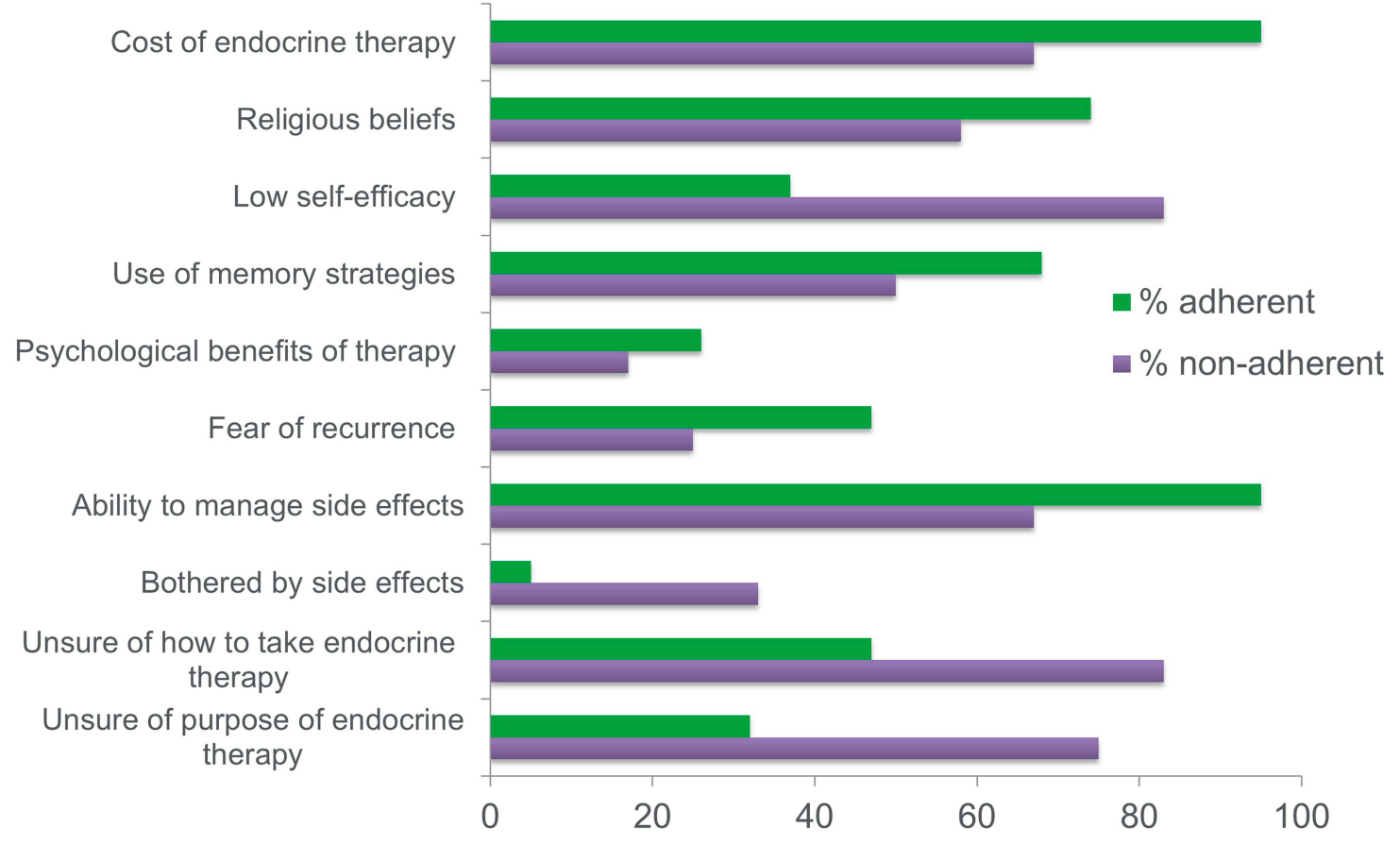

Patients classified as non-adherent defined per the threshold on the Revised Medication Adherence Questionnaire.

Figure 2 Facilitators and barriers to medication adherence identified in the concept elicitation study.

translatability review was conducted by a PROMIS linguist. Items with unclear or confusing wording and meaning were flagged, which resulted in minor revisions to the wording of several items. After this translatability review and revisions, the item set was translated into Italian.

The PMAS is flexible in its ability to ask either about one medication at a time or about an entire medication regimen, each specified by the user at the start of the assessment. In addition, to provide context for the nine core medication adherence items, there is an introductory question asking how often the specified medication is prescribed to be taken. Similarly, two follow-up items are included at the end of the measure to provide additional context. Both items were taken from the Functional Assessment of Chronic Illness Therapy (FACIT) measurement system regarding the severity of side effect burden ${ }^{59}$ and the extent the medication creates financial hardship. ${ }^{60}$ These items will not be included in the scoring for the PMAS but will provide additional information regarding the impact of side effects and costs of adherence to supplement the medication adherence items.

\section{Cognitive Interviews}

We recruited five patients to participate in the cognitive interviews. Three of these were diagnosed with diabetes, one was diagnosed with breast cancer, and one was the recipient of a kidney transplant. On average, they had been diagnosed 11 years prior to the time of the interview. Each of these patients was taking one or more oral medication for their health condition. Three of the five were male, were on average 57 years old, and were mostly White (four of five; fifth was Black or African American). Two of the five had achieved an advanced level of education (Master's, JD, PhD), one reported a college degree (undergraduate), one reported some college, and one reported a high school/GED education. Three of the five were employed full time, and two were retired. On the Eastern Cooperative Oncology Group (ECOG) performance status rating, two reported having normal activity with no symptoms (ECOG score 0), two reported some symptoms without requiring bed rest during the day (ECOG score 1), and one reported needing bed rest for less than $50 \%$ of the day (ECOG score 2).

Each of the patients commented that they fully understood the instructions. When asked to repeat the item in 
their own words, all participants indicated that they understood the intended item meaning and could critically appraise the item's clarity. Most of the items (8/12) required no changes. The final items, item names, and their response options are given in Table 3. Four total changes were made based on the cognitive interview results. Critically, these changes were limited to item phrasing, and no changes in the content areas were made based on the patients' input. In one case, a phrase was changed from "told to take this medicine" to "supposed to take this medicine" (MedAdFRQ). In another case, an item was reworded from "I did not take this medicine because I felt better" to "I stopped taking this medicine because I thought I did not need it" (MedAd7). Finally, for two items regarding the impact of medication side effects (MedAd7 and GP5), item-specific instructions were added to help guide response choices for cases when the patient has no side effects. Concurrently, cognitive interviews were held with Italian cancer patients to ensure that the items were comprehensible and culturally relevant in the Italian context.

\section{Discussion}

This paper describes the development of the NIH PROMIS Medication Adherence Scale (PMAS). There is a need for brief, patient-oriented, freely available, and clinicallyfeasible patient-reported medication adherence measures to be implemented in routine practice. To develop the PMAS, we undertook a multi-phase study that included reviewing and synthesizing previous systematic reviews of the literature, analyzing content areas from commonly used legacy measures, conducting a concept elicitation interview study with patients to identify additional key content areas to be addressed in the new measure, and extensive item revision, including cognitive interviews with a diverse set of patients with chronic illnesses. Then, we generated a new set of items to create the PMAS. With nine medication adherence items, this

Table 3 PROMIS Medication Adherence Scale Items

\begin{tabular}{|c|c|c|}
\hline $\begin{array}{l}\text { Item } \\
\text { Name }\end{array}$ & Wording & Response Options \\
\hline MedAdFRQ & $\begin{array}{l}\text { How many times per day are you supposed to take this } \\
\text { medicine? }\end{array}$ & $\begin{array}{l}\text { "Less than once per day", "Once per day", "Twice per day", "Three } \\
\text { or more per day" }\end{array}$ \\
\hline MedAdI & I know how to take this medicine as recommended. & $\begin{array}{l}\text { "Strongly disagree”, “Disagree”, “Neither agree or disagree”, } \\
\text { "Agree”, "Strongly agree” }\end{array}$ \\
\hline MedAd2 & I understand why I need to take this medicine. & $\begin{array}{l}\text { "Strongly disagree", "Disagree", "Neither agree or disagree”, } \\
\text { "Agree”, "Strongly agree" }\end{array}$ \\
\hline MedAd3 & I believe it is important to take this medicine. & $\begin{array}{l}\text { "Strongly disagree”, “Disagree”, “Neither agree or disagree”, } \\
\text { "Agree", "Strongly agree" }\end{array}$ \\
\hline MedAd4 & I believe this medicine is working. & $\begin{array}{l}\text { "Strongly disagree”, “Disagree”, “Neither agree or disagree”, } \\
\text { "Agree”, "Strongly agree” }\end{array}$ \\
\hline MedAd5 $5^{\mathrm{a}}$ & I took this medicine as recommended. & “Never”, “Rarely”, “Sometimes”, “Almost always”, “Always” \\
\hline MedAd6 $^{\mathrm{a}}$ & I remembered to take this medicine. & “Never”, “Rarely”, “Sometimes”, “Almost always”, “Always” \\
\hline MedAd7 $7^{\mathrm{a}, \mathrm{b}}$ & $\begin{array}{l}\text { I did not take this medicine because it caused side effects } \\
\text { that bothered me. }\end{array}$ & “Never”, “Rarely”, “Sometimes”, “Almost always”, “Always” \\
\hline MedAd8 ${ }^{\mathrm{a}}$ & $\begin{array}{l}\text { I stopped taking this medicine because I thought I did not } \\
\text { need it. }\end{array}$ & “Never”, “Rarely”, “Sometimes”, “Almost always”, “Always” \\
\hline MedAd9 ${ }^{a}$ & I did not take this medicine because of the cost. & "Never", “Rarely”, “Sometimes”, “Almost always”, “Always” \\
\hline $\mathrm{GP}^{\mathrm{a}, \mathrm{c}}$ & I am bothered by the side effects of treatment ... & "Not at all”, "A little bit", "Somewhat", "Quite a bit", "Very much" \\
\hline $\mathrm{FTI} 2^{\mathrm{a}}$ & $\begin{array}{l}\text { The cost of my medicine has been a financial hardship to my } \\
\text { family and me. }\end{array}$ & "Not at all”, "A little bit", "Somewhat", "Quite a bit", "Very much" \\
\hline
\end{tabular}

Notes: aAdministered with the context, "In the past 7 days ...". ' Administered with the item-specific instruction: "Note: Please select 'Never' if you have no side effects." 'Administered with the item-specific instruction: "Note: Please select 'Not at all' if you have no side effects." Items copyrighted by the authors; reprinted with permission. 
measure is brief but flexible (eg, able to accommodate many medications and can be used for different diseases) and clinically relevant.

Over the past several years, there has been a significant increase in emphasis on using PROs to enhance routine clinical monitoring and in a variety of research settings, including clinical trials. The majority of this effort has focused on outcomes of care like symptoms and HRQOL, and the PROMIS system has contributed many PRO measures to this effort. ${ }^{34}$ However, there is emerging recognition that other content areas critical to improving healthcare should be given the same rigorous treatment in their development as PROMIS has applied in the development of outcome measures. It is in this spirit that we developed a measure for medication adherence, a health behavior. Previous research has commented on potential biases emerging from patient-reports of behaviors like medication taking, especially in comparison to objective methods for measuring this behavior, like electronic pill caps. ${ }^{61,62}$ In addition, others have argued that patient-reported medication adherence measures that include questions related to adherence barriers, beliefs, and knowledge introduce heterogeneous content that does not strictly measure medication adherence. $^{46}$ We argue that the benefits of patient-reported medication adherence measurement outweigh these issues. Key benefits include easy implementation, ability to administer the measure in a discussion-based or conversational manner, ability to identify reasons for non-adherence in clinical counselling, brevity, and low cost. ${ }^{46,63,64}$ In addition, because it was informed by qualitative work with patients, the PMAS reflects elements of medication adherence that patients find most important. Our cognitive interviews then confirmed the relevance of PMAS's items to patients' medication experiences.

This manuscript details the steps taken to develop the PMAS, but there are several important next steps needed to examine its measurement properties. First, psychometric analyses of the items' dimensionality will help determine whether the items represent one or more constructs related to adherence (eg, extent of adherence, barriers to adherence). In turn, the results of these analyses will inform the approach to scoring. Next, we are planning multiple validation studies that will examine whether the PMAS is associated with other measures of medication adherence. The most useful test of validity for patient-reported medication adherence measures is likely criterion validitytesting the self-report measure against the criterion of medication adherence measured with electronic pill caps or biological measures (eg, presence of medication in the blood). ${ }^{46}$ In addition, more-so than other patient-reported measures, receiver-operator characteristic (ROC) curve analysis to determine positive predictive value, negative predictive value, sensitivity, and specificity are important for patient-reported adherence measures. These analyses will be the focus of future validation studies.

Particular aspects of the PROMIS approach to measure development and validation may also be applied to the PMAS in future work. Of particular note is the use of item response theory (IRT) to estimate useful item properties. IRT is a family of statistical models that can be used to directly estimate the level of the underlying trait or concept being measured for each individual (eg, an individual's level of medication adherence). IRT has recently been applied to health measurement with great success. ${ }^{65-67}$ This is in contrast to classical test theory (CTT), which can only determine an individual's trait level after summing (or otherwise combining) an individual's item responses. Most patient-reported medication adherence scales are based on CTT and use this method to estimate an individual's level of adherence. IRT models can also be used to determine an item's difficulty (eg, at what level of medication adherence will a person be likely to give a particular response) and ability to discriminate between individuals of higher and lower levels of medication adherence. These item properties can be used to determine which items make the best clinical screeners or give most information about patients' adherence behaviors. Finally, using these item properties, measures using IRT can be administered in computer adaptive tests, which facilitates very brief but highly reliable assessments. PROMIS measures have taken advantage of these strong capabilities within IRT to create gold standard measures of HRQOL and symptoms that have proved very useful in clinical screening with validity across many chronic conditions. ${ }^{34,68,69}$ In future phases of development and validation, we will consider applying IRT to the PMAS as well. It is worth noting that the PMAS is unlikely to use one IRT-oriented property common to many PROMIS measures, which is the use of norm-based scores to provide easy reference to a comparison population, like the US general population. ${ }^{70}$ Due to the significant variation in medications and their use across many diverse chronic conditions, it is unlikely that there are useful, general, normative population values of medication adherence.

This paper has important limitations to consider. First, this paper reported only on the PMAS's development, and no quantitative validation data for the PMAS are available at this date. For this reason, potential users should be cautious about implementing this measure until such data are available. As we have detailed above, plans for multiple validation studies are ongoing in a diverse array of samples with respect to 
health condition, as well as demographic characteristics. In addition, although we were able to draw upon legacy measures relevant to multiple clinical areas and patient input for selecting the content areas to be measured in the PMAS from patients with cancer (concept elicitation and cognitive interviews), diabetes (cognitive interviews), and kidney transplant recipients (cognitive interviews), the conclusions are drawn from patients with these conditions may not be applicable to all conditions, and additional qualitative data should be sought to add to the current data and to cover a broader range of chronic conditions. Likewise, though our cognitive interviews were able to gauge the items' clarity, additional issues may be caught after larger-scale administration in quantitative studies, necessitating future amendments to improve the items. Measure development and validation are an iterative process, and the demands are high on PROMIS measures to establish wide-ranging clinical applicability. We are currently conducting additional qualitative work with patients to learn more about the content areas most relevant for inclusion in the PMAS.

In conclusion, the PMAS is a brief and useful patientreported measure of medication adherence that will be appropriate for clinical adherence screening and research and that will be free for use to the public. We have used rigorous standards for measure development, triangulating between multiple sources of information to determine which content areas were most relevant to measuring medication adherence, and writing a new set of items that were subjected to thorough reviews for comprehension and translatability. In the next phases of the project, we will conduct international field-testing and quantitative evaluation of the final set of PMAS items through several validation studies, including an ongoing study using the Italian version being conducted in Rome, Italy. The final version of the PMAS will be freely available to researchers and clinicians for use in research and clinical care.

\section{Ethical Approval}

The data used in this study were collected under studyspecific protocols and are not freely available. The Northwestern University Institutional Review Board (IRB) reviewed these protocols. After review, both the concept elicitation study and cognitive interview study were given non-human subjects exemption (\#STU00210027). Despite receipt of non-human subjects exemption, written consent was obtained before participation in each study.

\section{Acknowledgments}

John Devin Peipert and Sherif M. Badawy are co-first authors for this study. We thank Helena Correia for translatability review of the PMAS.

\section{Disclosure}

Dr John Devin Peipert reports grants from Pfizer, Bristol Myers Squibb, and Veloxis Pharmaceuticals and personal fees from AstraZeneca, outside the submitted work. Dr Fabio Efficace reports personal fees from Amgen, Bristol Myers Squibb, Orsenix, Incyte, Takeda, grants from Amgen, outside the submitted work. Dr Daniel K Mroczek reports personal fees from International Drug Development Institute, outside the submitted work. Dr Michael Wolf reports grants from Amgen, grants, personal fees from Merck, Sharpe \& Dohme, Pfizer, personal fees from Sanofi, Luto UK, grants from Eli Lilly, outside the submitted work. Dr David Cella reports grants from the National Institutes of Health, during the conduct of the study. Dr Laura B Oswald is now affiliated with Health Outcomes and Behavior Program, Moffitt Cancer Center. The authors report no other conflicts of interest in this work.

\section{References}

1. Cheen MHH, Tan YZ, Oh LF, Wee HL, Thumboo J. Prevalence of and factors associated with primary medication non-adherence in chronic disease: a systematic review and meta-analysis. Int $J$ Clin Pract. 2019;73(6):e13350. doi:10.1111/ijcp.13350

2. Conn VS, Enriquez M, Ruppar TM, Chan KC. Meta-analyses of theory use in medication adherence intervention research. $\mathrm{Am}$ $J$ Health Behav. 2016;40(2):155-171. doi:10.5993/AJHB.40.2.1

3. Conn VS, Ruppar TM. Medication adherence outcomes of 771 intervention trials: systematic review and meta-analysis. Prev Med. 2017;99:269-276. doi:10.1016/j.ypmed.2017.03.008

4. Conn VS, Ruppar TM, Enriquez M, Cooper P. Medication adherence interventions that target subjects with adherence problems: systematic review and meta-analysis. Res Social Adm Pharm. 2016;12 (2):218-246. doi:10.1016/j.sapharm.2015.06.001

5. Gast A, Mathes T. Medication adherence influencing factors-an (updated) overview of systematic reviews. Syst Rev. 2019;8(1):112. doi:10.1186/s13643-019-1014-8

6. Christiansen AJ. Patient Adherence to Medical Treatment Regimens. New Heaven, CT: Yale University Press; 2004.

7. Organization WH. Adherence to long-term therapies: evidence for action. World Health Organization; 2003. Available from: https:// www.who.int/chp/knowledge/publications/adherence_report/en/. Accessed June 10, 2019.

8. Osterberg L, Blaschke T. Adherence to medication. $N$ Engl $J$ Med. 2005;353(5):487-497.

9. Rapoff M. Adherence to pediatric medical regimens. In: Issues in Clinical Child Psychology. 2nd ed. New York: Springer Science +Business Media; 2010

10. Mira JJ, Orozco-Beltrán D, Pérez-Jover V, et al. Physician patient communication failure facilitates medication errors in older polymedicated patients with multiple comorbidities. Fam Pract. 2012;30 (1):56-63. doi:10.1093/fampra/cms046 
11. Bailey SC, Opsasnick LA, Curtis LM, et al. Longitudinal investigation of older adults' ability to self-manage complex drug regimens. $J$ Am Geriatr Soc. 2019. doi:10.1111/jgs. 16255

12. McDonald HP, Garg AX, Haynes RB. Interventions to enhance patient adherence to medication prescriptions: scientific review. JAMA. 2002;288(22):2868-2879. doi:10.1001/jama.288.22.2868

13. Urquhart J, De Klerk E. Contending paradigms for the interpretation of data on patient compliance with therapeutic drug regimens. Stat Med. 1998;17(3):251-267; discussion 387-259. doi:10.1002/(SICI) 1097-0258(19980215)17:3<251::AID-SIM762>3.0.CO;2-A

14. Cheetham TC, Niu F, Green K, et al. Primary nonadherence to statin medications in a managed care organization. J Manag Care Pharm. 2013;19(5):367-373. doi:10.18553/jmcp.2013.19.5.367

15. Latry P, Pinet M, Labat A, et al. Adherence to anti-inflammatory treatment for asthma in clinical practice in France. Clin Ther. 2008;30:Spec No:1058-1068. doi:10.1016/j.clinthera.2008.06.011

16. Badawy SM, Thompson AA, Penedo FJ, Lai J-S, Rychlik K, Liem RI. Barriers to hydroxyurea adherence and health-related quality of life in adolescents and young adults with sickle cell disease. Eur J Haematol. 2017;98(6):608-614.

17. Badawy SM, Thompson AA, Liem RI. Beliefs about hydroxyurea in youth with sickle cell disease. Hematol Oncol Stem Cell Ther. 2018;11(3):142-148. doi:10.1016/j.hemonc.2018.01.001

18. Jackevicius CA, Li P, Tu JV. Prevalence, predictors, and outcomes of primary nonadherence after acute myocardial infarction. Circulation. 2008;117(8):1028-1036. doi:10.1161/CIRCULATIONAHA.107.706 820

19. Lee JS, Joyce G, McCombs J. Outcomes associated with primary and secondary nonadherence to cholesterol medications. Am J Pharm Benefits. 2016;8(2):54-60.

20. Simpson SH, Eurich DT, Majumdar SR, et al. A meta-analysis of the association between adherence to drug therapy and mortality. BMJ. 2006;333(7557):15. doi:10.1136/bmj.38875.675486.55

21. Badawy SM, Thompson AA, Lai JS, Penedo FJ, Rychlik K, Liem RI. Health-related quality of life and adherence to hydroxyurea in adolescents and young adults with sickle cell disease. Pediatr Blood Cancer. 2017;64(6):e26369. doi:10.1002/pbc.26369

22. DiMatteo MR, Giordani PJ, Lepper HS, Croghan TW. Patient adherence and medical treatment outcomes: a meta-analysis. Med Care. 2002;40(9):794-811. doi:10.1097/00005650-200209000-00009

23. Europe Fo. Just what the doctor ordered: an EU response to medication nonadherence. Friends of Europe; 2010. Available from: https:// www.friendsofeurope.org/. Accessed June 11, 2019.

24. Efficace F, Rosti G, Cottone F, et al. Profiling chronic myeloid leukemia patients reporting intentional and unintentional non-adherence to lifelong therapy with tyrosine kinase inhibitors. Leuk Res. 2014;38(3):294-298. doi:10.1016/j.leukres.2013.07.003

25. Badawy SM, Thompson AA, Kuhns LM. Medication adherence and technology-based interventions for adolescents with chronic health conditions: a few key considerations. JMIR Mhealth Uhealth. 2017;5 (12):e202. doi: $10.2196 /$ mhealth. 8310

26. Badawy SM, Barrera L, Sinno MG, Kaviany S, O’Dwyer LC, Kuhns LM. Text messaging and mobile phone apps as interventions to improve adherence in adolescents with chronic health conditions: a systematic review. JMIR Mhealth Uhealth. 2017;5(5):e66. doi:10.2196/mhealth. 7798

27. Garfield S, Clifford S, Eliasson L, Barber N, Willson A. Suitability of measures of self-reported medication adherence for routine clinical use: a systematic review. BMC Med Res Methodol. 2011;11(1):149. doi:10.1186/1471-2288-11-149

28. Lam WY, Fresco P. Medication adherence measures: an overview. Biomed Res Int. 2015;2015:12. doi:10.1155/2015/217047

29. Cleemput I, Dobbels F. Measuring patient-reported outcomes in solid organ transplant recipients. PharmacoEconomics. 2007;25 (4):269-286. doi:10.2165/00019053-200725040-00002
30. Nguyen T-M-U, Caze AL, Cottrell N. What are validated self-report adherence scales really measuring?: a systematic review. Br J Clin Pharmacol. 2014;77(3):427-445. doi:10.1111/bcp.12194

31. Kim C-J, Schlenk EA, Ahn J-A, Kim M, Park E, Park J. Evaluation of the measurement properties of self-reported medication adherence instruments among people at risk for metabolic syndrome: a systematic review. Diabetes Educ. 2016;42(5):618-634. doi:10.11 $77 / 0145721716655400$

32. Morisky DE, Green LW, Levine DM. Concurrent and predictive validity of a self-reported measure of medication adherence. Med Care. 1986;24(1):67-74. doi:10.1097/00005650-198601000-00007

33. Morisky DE, Ang A, Krousel-Wood M, Ward HJ. Predictive validity of a medication adherence measure in an outpatient setting. J Clin Hypertens (Greenwich). 2008;10(5):348-354. doi:10.1111/j.17517176.2008.07572.x

34. Cella D, Riley W, Stone A, et al. The Patient-Reported Outcomes Measurement Information System (PROMIS) developed and tested its first wave of adult self-reported health outcome item banks: 2005-2008. J Clin Epidemiol. 2010;63(11):1179-1194. doi:10.1016/ j.jclinepi.2010.04.011

35. Cella D, Yount S, Rothrock N, et al. The Patient-Reported Outcomes Measurement Information System (PROMIS): progress of an $\mathrm{NIH}$ Roadmap cooperative group during its first two years. Med Care. 2007;45(5 Suppl 1):S3-S11. doi:10.1097/01.mlr.0000258615.42478.55

36. DeWalt DA, Rothrock N, Yount S, Stone AA, Group PC. Evaluation of item candidates: the PROMIS qualitative item review. Med Care. 2007;45(5 Suppl 1):S12-S21. doi:10.1097/01.mlr.0000254567.79 743.e2

37. Hahn EA, Devellis RF, Bode RK, et al. Measuring social health in the patient-reported outcomes measurement information system (PROMIS): item bank development and testing. Qual Life Res. 2010;19(7):1035-1044. doi:10.1007/s11136-010-9654-0

38. Reeve BB, Hays RD, Bjorner JB, et al. Psychometric evaluation and calibration of health-related quality of life item banks: plans for the Patient-Reported Outcomes Measurement Information System (PROMIS). Med Care. 2007;45(5 Suppl 1):S22-S31. doi:10.1097/ 01.mlr.0000250483.85507.04

39. PROMIS Cooperative Group. PROMIS ${ }^{\circledR}$ instrument development and validation scientific standards version 2.0; 2013. Available from: http:// www.healthmeasures.net/images/PROMIS/PROMISStandards_Vers2.0_ Final.pdf. Accessed May 11, 2019, 2019.

40. Cella D, Choi SW, Condon DM, et al. PROMIS((R)) adult health profiles: efficient short-form measures of seven health domains. Value Health. 2019;22(5):537-544. doi:10.1016/j.jval.2019.02.004

41. Chlebowski RT, Kim J, Haque R. Adherence to endocrine therapy in breast cancer adjuvant and prevention settings. Cancer Prev Res. 2014;7(4):378. doi:10.1158/1940-6207.CAPR-13-0389

42. Lehmann A, Aslani P, Ahmed R, et al. Assessing medication adherence: options to consider. Int J Clin Pharm. 2014;36(1):55-69. doi:10.1007/s11096-013-9865-x

43. Bissonnette JM. Adherence: a concept analysis. $J$ Adv Nurs. 2008;63 (6):634-643. doi:10.1111/j.1365-2648.2008.04745.x

44. Culig J, Leppée M. From Morisky to hill-bone; self-reports scales for measuring adherence to medication. Coll Antropol. 2014;38 (1):55-62.

45. Lavsa SM, Holzworth A, Ansani NT. Selection of a validated scale for measuring medication adherence. J Am Pharm Assoc. 2011;51 (1):90-94. doi:10.1331/JAPhA.2011.09154

46. Stirratt MJ, Dunbar-Jacob J, Crane HM, et al. Self-report measures of medication adherence behavior: recommendations on optimal use. Transl Behav Med. 2015;5(4):470-482. doi:10.1007/s13142-015$0315-2$

47. Murray MD, Morrow DG, Weiner M, et al. A conceptual framework to study medication adherence in older adults. Am J Geriatr Pharmacother. 2004;2(1):36-43. doi:10.1016/S1543-5946(04)90005-0 
48. Voils CI, Hoyle RH, Thorpe CT, Maciejewski ML, Yancy WS Jr. Improving the measurement of self-reported medication nonadherence. J Clin Epidemiol. 2011;64(3):250-254. doi:10.1016/j.jclinepi.2010.07. 014

49. Matza LS, Park J, Coyne KS, Skinner EP, Malley KG, Wolever RQ. Derivation and validation of the ASK-12 adherence barrier survey. Ann Pharmacother. 2009;43(10):1621-1630. doi:10.1345/aph.1M174

50. Wu JR, Chung M, Lennie TA, Hall LA, Moser DK. Testing the psychometric properties of the medication adherence scale in patients with heart failure. Heart Lung. 2008;37(5):334-343. doi:10.1016/j. hrtlng.2007.10.001

51. Zeller A, Schroeder K, Peters TJ. An adherence self-report questionnaire facilitated the differentiation between nonadherence and nonresponse to antihypertensive treatment. J Clin Epidemiol. 2008;61 (3):282-288. doi:10.1016/j.jclinepi.2007.04.007

52. Breuil V, Cortet B, Cotte FE, et al. Validation of the adherence evaluation of osteoporosis treatment (ADEOS) questionnaire for osteoporotic post-menopausal women. Osteoporos Int. 2012;23 (2):445-455. doi:10.1007/s00198-011-1555-8

53. Feldman BJ, Fredericksen RJ, Crane PK, et al. Evaluation of the single-item self-rating adherence scale for use in routine clinical care of people living with HIV. AIDS Behav. 2013;17(1):307-318. doi:10.1007/s10461-012-0326-7

54. Knobel H, Alonso J, Casado JL, et al. Validation of a simplified medication adherence questionnaire in a large cohort of HIV-infected patients: the GEEMA Study. AIDS. 2002;16(4):605-613. doi:10.1097/00002030-200203080-00012

55. Chisholm MA, Lance CE, Williamson GM, Mulloy LL. Development and validation of the immunosuppressant therapy adherence instrument (ITAS). Patient Educ Couns. 2005;59(1):13-20.

56. Kripalani S, Risser J, Gatti ME, Jacobson TA. Development and evaluation of the Adherence to Refills and Medications Scale (ARMS) among low-literacy patients with chronic disease. Value Health. 2009;12(1):118-123. doi:10.1111/j.1524-4733.2008.00400.x

57. Voils CI, Maciejewski ML, Hoyle RH, et al. Initial validation of a self-report measure of the extent of and reasons for medication nonadherence. Med Care. 2012;50(12):1013-1019. doi:10.1097/ MLR.0b013e318269e121

58. Gruber-Baldini AL, Velozo C, Romero S, Shulman LM. Validation of the PROMIS((R)) measures of self-efficacy for managing chronic conditions. Qual Life Res. 2017;26(7):1915-1924. doi:10.1007/ s11136-017-1527-3
59. Pearman TP, Beaumont JL, Mroczek D, O’Connor M, Cella D Validity and usefulness of a single-item measure of patient-reported bother from side effects of cancer therapy. Cancer. 2018;124 (5):991-997. doi:10.1002/cncr.31133

60. de Souza JA, Yap BJ, Hlubocky FJ, et al. The development of a financial toxicity patient-reported outcome in cancer: the COST measure. Cancer. 2014;120(20):3245-3253.

61. Grymonpre RE, Didur CD, Montgomery PR, Sitar DS. Pill count, self-report, and pharmacy claims data to measure medication adherence in the elderly. Ann Pharmacother. 1998;32(7-8):749-754.

62. Daniels T, Goodacre L, Sutton C, Pollard K, Conway S, Peckham D. Accurate assessment of adherence: self-report and clinician report vs electronic monitoring of nebulizers. Chest. 2011;140(2):425-432. doi:10.1378/chest.09-3074

63. Myaskovsky L, Jesse MT, Kuntz K, et al. Report from the American Society of Transplantation Psychosocial Community of Practice Adherence Task Force: real-world options for promoting adherence in adult recipients. Clin Transplant. 2018;32(9):e13353.

64. Maldonado AQ, West-Thielke P, Dew MA, et al. Meeting report: consensus recommendations for a research agenda to address immunosuppressant nonadherence in organ transplantation. Clin Transplant. 2018;32(9):e13362.

65. Edelen MO, Reeve BB. Applying item response theory (IRT) modeling to questionnaire development, evaluation, and refinement. Qual Life Res. 2007;16:5-18.

66. Hays RD, Morales LS, Reise SP. Item response theory and health outcomes measurement in the 21st century. Med Care. 2000;38(9 Suppl):II28-II42.

67. Cappelleri JC, Jason Lundy J, Hays RD. Overview of classical test theory and item response theory for the quantitative assessment of items in developing patient-reported outcomes measures. Clin Ther. 2014;36(5):648-662.

68. Fries JF, Bruce B, Cella D. The promise of PROMIS: using item response theory to improve assessment of patient-reported outcomes. Clin Exp Rheumatol. 2005;23:S53.

69. Schalet BD, Hays RD, Jensen SE, Beaumont JL, Fries JF, Cella D. Validity of PROMIS physical function measured in diverse clinical samples. J Clin Epidemiol. 2016;73:112-118.

70. Liu H, Cella D, Gershon R, et al. Representativeness of the patient-reported outcomes measurement information system internet panel. J Clin Epidemiol. 2010;63(11):1169-1178.
Patient Preference and Adherence

\section{Publish your work in this journal}

Patient Preference and Adherence is an international, peer-reviewed, open access journal that focuses on the growing importance of patient preference and adherence throughout the therapeutic continuum. Patient satisfaction, acceptability, quality of life, compliance, persistence and their role in developing new therapeutic modalities and compounds to optimize clinical outcomes for existing disease states are major areas of interest for the journal. This journal has been accepted for indexing on PubMed Central. The manuscript management system is completely online and includes a very quick and fair peer-review system, which is all easy to use. Visit http:// www.dovepress.com/testimonials.php to read real quotes from published authors. 